\title{
Intestinal parasitic infections and associated factors among pregnant women in Ethiopia: a systematic review and meta- analysis
}

Zelalem Animaw ${ }^{1 *}$, Addisu Melese ${ }^{2}$, Habtamu Demelash ${ }^{3}$, Girma Seyoum ${ }^{4}$ and Abiy Abebe ${ }^{5}$

\begin{abstract}
Background: Intestinal parasitic infections (IPIs) are public health problems widely distributed in the world and cause significant morbidity and mortality; many of which occur among women of reproductive age. IPIs caused by helminthes and protozoan parasites are common among pregnant women. Data on the national pooled prevalence of intestinal parasites and associated factors during pregnancy is not documented well in Ethiopia. This review aims at summarizing evidences on the burden of IPIs and associated factors among pregnant women in Ethiopia.

Methods: Published and unpublished studies were thoroughly searched at MEDLINE/PubMed, EMBASE, Google Scholar, CINAHL, Cochrane library and Science Direct. In addition, repositories of Addis Ababa, Gondar and Jimma Universities were searched. Eligible studies were selected following the Preferred Reporting Items for Systematic Reviews and Meta-Analysis (PRISMA) guideline. The pooled prevalence of intestinal parasites and summary odds ratios (ORs) were determined with $95 \%$ confidence intervals (Cl). Sub-groups analyses were done based on study region, types of parasites, methods of stool examination and study setting. The statistical analyses were performed using STATA version 14.0 software.
\end{abstract}

Results: Among 168 retrieved studies, 31 studies with a total population of 12,118 pregnant women were included. The estimated pooled prevalence of IPIs among pregnant women in Ethiopia was 27.32\% (95 \% Cl: 20.61, 33.87\%). In the subgroup analysis, Oromia and Amhara regions had the highest prevalence with a $29.78 \%$ (95 \% Cl: 15.97 , 43.60) and $29.63 \%$ (95\% Cl: 15.37, 43.89); respectively. In addition, studies conducted in the community showed higher prevalence than institution based studies (49.93\% Vs $24.84 \%$; respectively). The most prevalent type of intestinal parasite identified were Hookworm followed by Ascaris lumbricoides with a pooled prevalence of 11.2 and $10.34 \%$, respectively. In our analysis; residence, being bare footed, lack of hand washing habit and eating uncooked/raw vegetables were significantly associated with IPIs among pregnant women in Ethiopia.

\footnotetext{
* Correspondence: zelalem.a01@gmail.com

'Department of Biomedical Sciences, College of Health Sciences, Debre

Tabor University, Debre Tabor, Ethiopia

Full list of author information is available at the end of the article
}

(c) The Author(s). 2021 Open Access This article is licensed under a Creative Commons Attribution 4.0 International License, which permits use, sharing, adaptation, distribution and reproduction in any medium or format, as long as you give appropriate credit to the original author(s) and the source, provide a link to the Creative Commons licence, and indicate if changes were made. The images or other third party material in this article are included in the article's Creative Commons licence, unless indicated otherwise in a credit line to the material. If material is not included in the article's Creative Commons licence and your intended use is not permitted by statutory regulation or exceeds the permitted use, you will need to obtain permission directly from the copyright holder. To view a copy of this licence, visit http://creativecommons.org/licenses/by/4.0/. The Creative Commons Public Domain Dedication waiver (http://creativecommons.org/publicdomain/zero/1.0/) applies to the data made available in this article, unless otherwise stated in a credit line to the data. 
Conclusions: Prevalence of IPIs during pregnancy is relatively high in Ethiopia. Poor hygienic practices were identified as risk factors. Based on our finding, targeted preventive measures shall be considered so as to prevent morbidity and mortality due to IPIs.

Keywords: Intestinal parasites, pregnant women, systematic review, meta-analysis, pregnancy, Ethiopia

\section{Background}

IPIs are public health problems widely distributed throughout the world causing significant morbidity, many of which occur among women of reproductive age. Pregnant women are one of high-risk population for these infections [1]. IPIs caused by helminths and protozoan parasites are common among pregnant women and experience more severe infections [2]. However, the severity depends on different factors, including parasitic load, species, inter-pregnancy intervals, nutritional status, poor hygiene and lack of safe drinking water, climate, poverty, immunity status, and the presence of co-existing infections [2-8].

Physiological changes during pregnancy modify the maternal immunity that brings tolerance to the growing fetus and susceptibility to different infections. IPIs are common during pregnancy that aggravates the effect leading to "a double burden to carry" and causes serious problems to the mother as well as to the embryo/fetus $[1,9]$. Moreover, IPIs might cause anemia; induce deficiencies of iron, total energy, protein, folate and zinc all of which results in low pregnancy weight gain and intrauterine growth retardation (IUGR), greater risks of infection, low birth weight (LBW) and higher perinatal mortality rates $[1,10-13]$.

IPIs are reportedly identified as the leading causes of maternal mortality in developing countries especially in the tropics and subtropics $[9,14,15]$. A recent systematic review and Meta-analysis on global prevalence and associated risk factors of IPIs revealed that IPIs in pregnant women is high especially in low and middle income countries [16]. Many other studies were conducted to assess the burden of soil-transmitted helminths (STH) and their effect during pregnancy. Preventive chemotherapy (PC) was introduced as a control program in order to reduce the burden of the infection, but it has been neglected for at-risk women of reproductive ages. The overall coverage of PC was reported as less than $75 \%$ in Ethiopia [17] howing the possibility of the high burden of intestinal parasites. Although it is expected to be high, data on the national pooled prevalence of intestinal parasites and associated factors during pregnancy is not yet documented well in Ethiopia. Therefore; this systematic review and Meta-analysis aims at providing summarized evidence on the burden of IPIs and associated factors among pregnant women in Ethiopia.

\section{Methods}

Data Bases and Search strategy

Both Published and unpublished studies regarding the magnitude of IPIs and associated factors among pregnant women in Ethiopia were thoroughly searched by two authors (ZA and AM) at databases of MEDLINE/ PubMed, EMBASE, Google Scholar, CINAHL, Cochrane Library and Science Direct. Additionally, repositories of Addis Ababa University, University of Gondar and Jimma University were searched manually to get unpublished student research works related to the topic. Reference lists of eligible studies were checked to maximize the inclusion of relevant studies. The search was not bounded by year of publication. As a result, all articles published and/or reported up to 25th May, 2020 were included. The Preferred Reporting Items for Systematic Reviews and MetaAnalyses (PRISMA) checklist was strictly followed to select potential studies (Fig. 1).

"Prevalence" "Magnitude", "Epidemiology", "Intestinal Parasite", "Intestinal parasitosis", "Helmenthiasis", "Hookworm" "Ascaris lumbricoides OR A. lumbericoids", "Schistosomia Mansoni OR S. mansoni", "Giardia lamblia OR G. lamblia", "Strongyloides stercolaris OR S. stercolaris", "Entamoeba histolytica OR E. hisolytica", " Trichuris trichiura OR T. trichiura", "Hymenolepis nana OR H. nana", "Taenia species", "Enterobius vermicularis OR E. vermicularis", "Associated Factors", "Determinants" "Pregnant Women", "Pregnant Mother" and "Ethiopia" were the key searching terms employed independently and/ or in combination by Boolean operators: "OR" or/and "AND" to bring key concepts together to identify relevant papers. Particularly, studies from MEDL INE/PubMed database were searched by using the following Medical Science Heading (MeSH) terms: (("parasitic infection"[All Fields] OR "intestinal parasite"[All Fields]) OR ("intestinal diseases, parasitic/epidemiology" [MeSH Terms] OR "intestinal diseases, parasitic"[MeSH Terms])) AND (((("pregnant women" [All Fields] OR "pregnant mothers"[All Fields]) OR "pregnant mothers attending anc"[All Fields]) AND "pregnant women attending anc"[All Fields]) OR (("pregnant women"[MeSH Terms] OR "pregnant women/epidemiology"[MeSH Terms]) OR "mothers"[MeSH Terms]))) AND "Ethiopia“[All Fields]. EndNote X7 was also used to manage duplication of articles. 


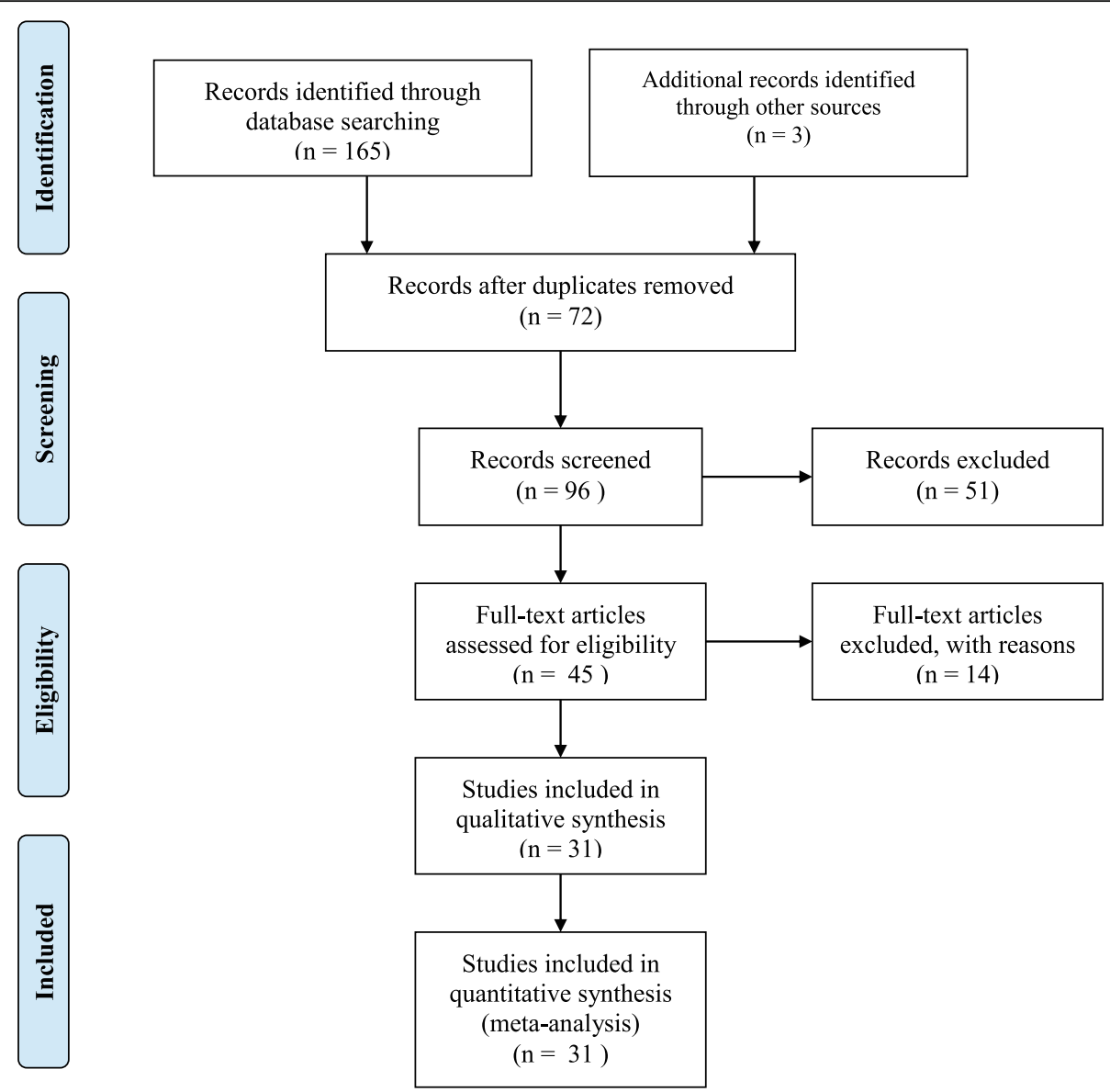

Fig. 1 PRISMA chart flow showing article selection process

\section{Inclusion criteria}

- Language of publication/written: English.

- Year of publication/report: Up to May 25th of 2020.

- Study design: observational studies (cross-sectional, case-control and cohort).

- Outcome: magnitude of IPIs and/ or associated factors.

- Study population: Pregnant women.

- Study setting: At (institution or community based).

- Study country: Ethiopia.

- Diagnostic modality: Stool examination (either wet mount or concentration or both).

- Type of parasite: Either protozoa or helminthes or both

- Types of articles: Both published and unpublished.

- Types of publication: Peer reviewed, full text articles.

\section{Exclusion criteria}

- Articles which failed to report the number of study participants and number of cases.
- Inaccessible full text articles due to nonresponsiveness of the corresponding authors upon frequent inquiry through email by two authors (ZA and AM).

- Systematic reviews and meta-analysis.

- Articles that did not provide calculable prevalence or ORs for associated factors.

\section{Data extraction}

Data pertaining authors' to name with publication year, study period, study design, study setting, study area/region, technique of stool examination, sample size, numbers of pregnant women infected with intestinal parasites and or prevalence of IPIs and species of parasites isolated were extracted from the eligible articles using Microsoft Excel 2013 sheet especially designed for this study. Similarly, a separate data extraction tool was developed for each identified associated factors which contain authors name and publication year. Two by wo tables were also employed to obtain the odds ratio from each study. All associated factors reported by at least two studies were included for pooled analysis. 
Table 1 General characteristic of the included articles for systematic review and meta-analysis pertaining magnitude and associated factors of IPIs among pregnant women in Ethiopia

\begin{tabular}{|c|c|c|c|c|c|c|c|c|c|}
\hline $\begin{array}{l}\text { Author, } \\
\text { publication } \\
\text { year }\end{array}$ & $\begin{array}{l}\text { Study } \\
\text { period }\end{array}$ & $\begin{array}{l}\text { Study } \\
\text { design }\end{array}$ & $\begin{array}{l}\text { Study } \\
\text { setting }\end{array}$ & $\begin{array}{l}\text { Study area/ } \\
\text { Region }\end{array}$ & $\begin{array}{l}\text { Technique of } \\
\text { stool } \\
\text { examination }\end{array}$ & $\begin{array}{l}\text { Sample } \\
\text { size }\end{array}$ & Cases & $\begin{array}{l}\text { Prevalence } \\
(\%)\end{array}$ & Parasite species isolated \\
\hline $\begin{array}{l}\text { Feleke and } \\
\text { Jember, } 2018 \\
{[20]}\end{array}$ & $\begin{array}{l}\text { November } \\
2015 \text { to } \\
\text { January } 2016\end{array}$ & $\begin{array}{l}\text { cross- } \\
\text { sectional }\end{array}$ & $\begin{array}{l}\text { Community } \\
\text { based }\end{array}$ & Mecha, Amhara & $\begin{array}{l}\text { formalin-ether } \\
\text { Concentration }\end{array}$ & 783 & 595 & 76.0 & $\begin{array}{l}\text { A. lumbricoides, S. mansoni, Hookworm, S. } \\
\text { stercolaris }\end{array}$ \\
\hline $\begin{array}{l}\text { Bolka and } \\
\text { Gebremedhin, } \\
2019[23]\end{array}$ & $\begin{array}{l}\text { June and } \\
\text { July } 2018\end{array}$ & $\begin{array}{l}\text { cross- } \\
\text { sectional }\end{array}$ & $\begin{array}{l}\text { Institution } \\
\text { based }\end{array}$ & $\begin{array}{l}\text { Wondo Genet, } \\
\text { SNNPR }\end{array}$ & $\begin{array}{l}\text { formalin-ether } \\
\text { Concentration }\end{array}$ & 349 & 135 & 38.7 & $\begin{array}{l}\text { Hookworm, S. mansoni, A. lumbricoides, T. } \\
\text { trichiura, G. lamblia, E. histolytica, }\end{array}$ \\
\hline $\begin{array}{l}\text { Derso et al., } \\
2016[4]\end{array}$ & $\begin{array}{l}\text { November } \\
2013 \text { to } \\
\text { January } 2014\end{array}$ & $\begin{array}{l}\text { cross- } \\
\text { sectional }\end{array}$ & $\begin{array}{l}\text { Institution } \\
\text { based }\end{array}$ & $\begin{array}{l}\text { Felege Hiwot } \\
\text { Hospital, Amhara }\end{array}$ & $\begin{array}{l}\text { formalin-ether } \\
\text { Concentration }\end{array}$ & 384 & 121 & 31.5 & $\begin{array}{l}\text { Hookworm, S. mansoni, A. lumbricoides, S. } \\
\text { stercolaris, T. trichiura, G. lamblia, E. } \\
\text { histolytica, H. nana, Taenia species }\end{array}$ \\
\hline $\begin{array}{l}\text { Yesuf et al., } 2019 \\
\text { [24] }\end{array}$ & $\begin{array}{l}\text { April } 1 \text { to } \\
\text { May } 15 \\
2019\end{array}$ & $\begin{array}{l}\text { cross- } \\
\text { sectional }\end{array}$ & $\begin{array}{l}\text { Institution } \\
\text { based }\end{array}$ & $\begin{array}{l}\text { Four Health } \\
\text { centers in Lalo } \\
\text { Kile, Oromia }\end{array}$ & Both & 315 & 138 & 43.8 & $\begin{array}{l}\text { Hookworm, A. lumbricoides, S. stercolaris, } \\
\text { T. trichiura, G. lamblia }\end{array}$ \\
\hline $\begin{array}{l}\text { Alem et al., } 2013 \\
{[25]}\end{array}$ & $\begin{array}{l}\text { February } \\
\text { to May } 2011\end{array}$ & $\begin{array}{l}\text { cross- } \\
\text { sectional }\end{array}$ & $\begin{array}{l}\text { Institution } \\
\text { based }\end{array}$ & $\begin{array}{l}\text { Azezo Health } \\
\text { Center. Amhara }\end{array}$ & $\begin{array}{l}\text { formalin-ether } \\
\text { Concentration }\end{array}$ & 384 & 55 & 14.3 & $\begin{array}{l}\text { Hookworm, S. mansoni, A. lumbricoides, G. } \\
\text { lamblia, E. histolytica, H. nana, Taenia } \\
\text { species }\end{array}$ \\
\hline $\begin{array}{l}\text { Lebso et al., } \\
2017 \text { [26] }\end{array}$ & $\begin{array}{l}\text { May-June } \\
2015\end{array}$ & $\begin{array}{l}\text { cross- } \\
\text { sectional }\end{array}$ & $\begin{array}{l}\text { Community } \\
\text { based }\end{array}$ & Lemo, SNNPR & $\begin{array}{l}\text { Direct wet } \\
\text { mount }\end{array}$ & 504 & 161 & 31.9 & $\begin{array}{l}\text { Hookworm, T. trichiura, A. lumbricoides, } \\
\text { Taenia species }\end{array}$ \\
\hline $\begin{array}{l}\text { Kefiyalew et al., } \\
2014[12]\end{array}$ & $\begin{array}{l}\text { March to } \\
\text { June } 2013\end{array}$ & $\begin{array}{l}\text { cross- } \\
\text { sectional }\end{array}$ & $\begin{array}{l}\text { Institution } \\
\text { based }\end{array}$ & $\begin{array}{l}\text { Bisidimo } \\
\text { Hospital, Oromia }\end{array}$ & Both & 258 & 96 & 37.2 & Not reported \\
\hline $\begin{array}{l}\text { Bekele et al., } \\
2016 \text { [27] }\end{array}$ & $\begin{array}{l}\text { February } 16 \\
\text { to April } 8 \\
2015\end{array}$ & $\begin{array}{l}\text { cross- } \\
\text { sectional }\end{array}$ & $\begin{array}{l}\text { Institution } \\
\text { based }\end{array}$ & $\begin{array}{l}\text { Arba Minch } \\
\text { hospital, SNNPR }\end{array}$ & $\begin{array}{l}\text { formalin-ether } \\
\text { Concentration }\end{array}$ & 332 & 40 & 12.0 & $\begin{array}{l}\text { Hookworm, A. lumbricoides, G. lamblia, E. } \\
\text { histolytica, Taenia species }\end{array}$ \\
\hline $\begin{array}{l}\text { Gedefaw et al., } \\
2015 \text { [28] }\end{array}$ & $\begin{array}{l}\text { January to } \\
\text { March } 2014\end{array}$ & $\begin{array}{l}\text { cross- } \\
\text { sectional }\end{array}$ & $\begin{array}{l}\text { Institution } \\
\text { based }\end{array}$ & $\begin{array}{l}\text { Otona Hospital, } \\
\text { SNNPR }\end{array}$ & $\begin{array}{l}\text { Direct wet } \\
\text { mount }\end{array}$ & 363 & 69 & 19.0 & $\begin{array}{l}\text { Hookworm, S. mansoni, A. lumbricoides, T. } \\
\text { trichiura, G. lamblia, E. histolytica, E. } \\
\text { vermicularis }\end{array}$ \\
\hline Asrie, 2017 [10] & $\begin{array}{l}\text { January to } \\
\text { March } 2015\end{array}$ & $\begin{array}{l}\text { cross- } \\
\text { sectional }\end{array}$ & $\begin{array}{l}\text { Institution } \\
\text { based }\end{array}$ & $\begin{array}{l}\text { Aymiba health } \\
\text { center, Amhara }\end{array}$ & $\begin{array}{l}\text { Direct wet } \\
\text { mount }\end{array}$ & 206 & 16 & 7.8 & Hookworm, E. vermicularis, A. lumbricoides, \\
\hline $\begin{array}{l}\text { Getahun et al., } \\
2017 \text { [29] }\end{array}$ & $\begin{array}{l}\text { March 01- } \\
\text { April } 30 \\
2015\end{array}$ & $\begin{array}{l}\text { cross- } \\
\text { sectional }\end{array}$ & $\begin{array}{l}\text { Institution } \\
\text { based }\end{array}$ & $\begin{array}{l}\text { Butajira hospital, } \\
\text { SNNPR }\end{array}$ & Both & 217 & 28 & 12.9 & Not reported \\
\hline $\begin{array}{l}\text { Ejeta et al., } 2014 \\
\text { [5] }\end{array}$ & $\begin{array}{l}\text { April to May, } \\
2014\end{array}$ & $\begin{array}{l}\text { cross- } \\
\text { sectional }\end{array}$ & $\begin{array}{l}\text { Institution } \\
\text { based }\end{array}$ & $\begin{array}{l}\text { Nekemte } \\
\text { Hospital, Oromia }\end{array}$ & $\begin{array}{l}\text { Direct wet } \\
\text { mount }\end{array}$ & 286 & 22 & 7.7 & Not reported \\
\hline $\begin{array}{l}\text { Zekarias et al., } \\
2017 \text { [43] }\end{array}$ & $\begin{array}{l}\text { April } 3 \text { to } \\
\text { May 3, } 2017\end{array}$ & $\begin{array}{l}\text { cross- } \\
\text { sectional }\end{array}$ & $\begin{array}{l}\text { Institution } \\
\text { based }\end{array}$ & $\begin{array}{l}\text { Mizan Tepi } \\
\text { hospital, SNNPR }\end{array}$ & $\begin{array}{l}\text { Direct wet } \\
\text { mount }\end{array}$ & 306 & 70 & 22.9 & Not reported \\
\hline $\begin{array}{l}\text { Melku et al., } \\
2014 \text { [38] }\end{array}$ & $\begin{array}{l}\text { March } 1 \text { to } \\
\text { April 30, } \\
2012\end{array}$ & $\begin{array}{l}\text { cross- } \\
\text { sectional }\end{array}$ & $\begin{array}{l}\text { Institution } \\
\text { based }\end{array}$ & $\begin{array}{l}\text { Gondar } \\
\text { University } \\
\text { Hospital Amhara }\end{array}$ & $\begin{array}{l}\text { Direct wet } \\
\text { mount }\end{array}$ & 302 & 80 & 26.5 & Hookworm, E. histolytica, A. lumbricoides, \\
\hline $\begin{array}{l}\text { Tesfaye, } 2015 \\
\text { [42] }\end{array}$ & $\begin{array}{l}\text { October } 1 \text { to } \\
30,2013\end{array}$ & $\begin{array}{l}\text { cross- } \\
\text { sectional }\end{array}$ & $\begin{array}{l}\text { Institution } \\
\text { based }\end{array}$ & $\begin{array}{l}\text { Nigist Eleni } \\
\text { hospital, SNNPR }\end{array}$ & $\begin{array}{l}\text { formalin-ether } \\
\text { Concentration }\end{array}$ & 258 & 76 & 29.5 & $\begin{array}{l}\text { Hookworm, T. trichiura, A. lumbricoides, } H \text {. } \\
\text { nana, Taenia species }\end{array}$ \\
\hline $\begin{array}{l}\text { Mengist et al., } \\
2017 \text { [13] }\end{array}$ & $\begin{array}{l}\text { November } \\
2015 \text { and } \\
\text { January } 2016\end{array}$ & $\begin{array}{l}\text { cross- } \\
\text { sectional }\end{array}$ & $\begin{array}{l}\text { Institution } \\
\text { based }\end{array}$ & $\begin{array}{l}\text { Five Health } \\
\text { Centers in } \\
\text { Wollega, Oromia }\end{array}$ & Both & 372 & 92 & 24.7 & $\begin{array}{l}\text { Hookworm, S. stercolaris, A. lumbricoides, } \\
\text { H. nana, Taenia species }\end{array}$ \\
\hline $\begin{array}{l}\text { Getachew et al., } \\
2013 \text { [32] }\end{array}$ & $\begin{array}{l}\text { August to } \\
\text { September, } \\
2011\end{array}$ & $\begin{array}{l}\text { cross- } \\
\text { sectional }\end{array}$ & $\begin{array}{l}\text { Community } \\
\text { based }\end{array}$ & $\begin{array}{l}\text { Gilge Gibe, } \\
\text { Oromia }\end{array}$ & $\begin{array}{l}\text { formalin-ether } \\
\text { Concentration }\end{array}$ & 388 & 162 & 41.8 & $\begin{array}{l}\text { Hookworm, T. trichiura, H. nana, E. } \\
\text { vermicularis, A. lumbricoides, }\end{array}$ \\
\hline Tefera. 2014 [41] & $\begin{array}{l}\text { April, } 1 \text { - } \\
\text { June 30, } \\
2014\end{array}$ & $\begin{array}{l}\text { cross- } \\
\text { sectional }\end{array}$ & $\begin{array}{l}\text { Institution } \\
\text { based }\end{array}$ & $\begin{array}{l}\text { Sher-Ethiopia } \\
\text { hospital, Oromia }\end{array}$ & Both & 748 & 436 & 58.3 & $\begin{array}{l}\text { A. lumbricoides, T. trichuria, Hook worm, } \\
\text { S. mansoni }\end{array}$ \\
\hline $\begin{array}{l}\text { Kumera et al., } \\
2018 \text { [36] }\end{array}$ & $\begin{array}{l}\text { July to } \\
\text { August } 2016\end{array}$ & $\begin{array}{l}\text { cross- } \\
\text { sectional }\end{array}$ & $\begin{array}{l}\text { Institution } \\
\text { based }\end{array}$ & $\begin{array}{l}\text { Debre Markos } \\
\text { Hospital, Amhara }\end{array}$ & Both & 234 & 64 & 27.4 & $\begin{array}{l}\text { Hookworm, E. histolytica } \\
\text { A. lumbricoides, G. lamblia, }\end{array}$ \\
\hline $\begin{array}{l}\text { Kebede et al., } \\
2018[22]\end{array}$ & $\begin{array}{l}\text { April 1-30, } \\
2018\end{array}$ & $\begin{array}{l}\text { cross- } \\
\text { sectional }\end{array}$ & $\begin{array}{l}\text { Institution } \\
\text { based }\end{array}$ & $\begin{array}{l}\text { Suhul hospital, } \\
\text { Tigray }\end{array}$ & $\begin{array}{l}\text { Not } \\
\text { mentioned }\end{array}$ & 480 & 35 & 7.3 & Not reported \\
\hline $\begin{array}{l}\text { Berhe et al., } \\
2019 \text { [30] }\end{array}$ & $\begin{array}{l}\text { April to } \\
\text { September } \\
2018\end{array}$ & $\begin{array}{l}\text { cross- } \\
\text { sectional }\end{array}$ & $\begin{array}{l}\text { Institution } \\
\text { based }\end{array}$ & $\begin{array}{l}\text { Adigrat Hospital, } \\
\text { Tigray }\end{array}$ & $\begin{array}{l}\text { Direct wet } \\
\text { mount }\end{array}$ & 304 & 54 & 17.8 & E. histolytica, G. lamblia, \\
\hline $\begin{array}{l}\text { Helion Belay } \\
\text { et al., } 2020 \text { [34] }\end{array}$ & $\begin{array}{l}\text { January } 11 \\
\text { to February } \\
20,2017\end{array}$ & $\begin{array}{l}\text { cross- } \\
\text { sectional }\end{array}$ & $\begin{array}{l}\text { Institution } \\
\text { based }\end{array}$ & $\begin{array}{l}\text { Health centers in } \\
\text { Dembia, Amhara }\end{array}$ & $\begin{array}{l}\text { Not } \\
\text { mentioned }\end{array}$ & 685 & 158 & 23.1 & Not reported \\
\hline $\begin{array}{l}\text { Kenea et al., } \\
2018 \text { [35] }\end{array}$ & $\begin{array}{l}\text { January to } \\
\text { July } 2016\end{array}$ & $\begin{array}{l}\text { cross- } \\
\text { sectional }\end{array}$ & $\begin{array}{l}\text { Institution } \\
\text { based }\end{array}$ & $\begin{array}{l}\text { Mettu, Bedele, } \\
\text { and ,Darimu }\end{array}$ & $\begin{array}{l}\text { Not } \\
\text { mentioned }\end{array}$ & 416 & 32 & 7.7 & Not reported \\
\hline
\end{tabular}


Table 1 General characteristic of the included articles for systematic review and meta-analysis pertaining magnitude and associated factors of IPIs among pregnant women in Ethiopia (Continued)

\begin{tabular}{|c|c|c|c|c|c|c|c|c|c|}
\hline $\begin{array}{l}\text { Author, } \\
\text { publication } \\
\text { year }\end{array}$ & $\begin{array}{l}\text { Study } \\
\text { period }\end{array}$ & $\begin{array}{l}\text { Study } \\
\text { design }\end{array}$ & $\begin{array}{l}\text { Study } \\
\text { setting }\end{array}$ & $\begin{array}{l}\text { Study area/ } \\
\text { Region }\end{array}$ & $\begin{array}{l}\text { Technique of } \\
\text { stool } \\
\text { examination }\end{array}$ & $\begin{array}{l}\text { Sample } \\
\text { size }\end{array}$ & Cases & $\begin{array}{l}\text { Prevalence } \\
(\%)\end{array}$ & Parasite species isolated \\
\hline & & & & $\begin{array}{l}\text { hospitals, } \\
\text { Oromia }\end{array}$ & & & & & \\
\hline Fassil, 2016 [21] & $\begin{array}{l}\text { December } \\
2015 \text { to } \\
\text { February } \\
2016\end{array}$ & $\begin{array}{l}\text { cross- } \\
\text { sectional }\end{array}$ & $\begin{array}{l}\text { Institution } \\
\text { based }\end{array}$ & $\begin{array}{l}\text { Selam Health } \\
\text { Cener, Addis } \\
\text { Ababa }\end{array}$ & $\begin{array}{l}\text { Not } \\
\text { mentioned }\end{array}$ & 480 & 35 & 7.3 & Not reported \\
\hline $\begin{array}{l}\text { Shiferaw et al., } \\
2017 \text { [40] }\end{array}$ & $\begin{array}{l}\text { March to } \\
\text { June, } 2015\end{array}$ & $\begin{array}{l}\text { cross- } \\
\text { sectional }\end{array}$ & $\begin{array}{l}\text { Institution } \\
\text { based }\end{array}$ & $\begin{array}{l}\text { Anbesame } \\
\text { health center, } \\
\text { Amhara }\end{array}$ & $\begin{array}{l}\text { Direct wet } \\
\text { mount }\end{array}$ & 180 & 38 & 21.1 & Hookworm, S. mansoni, \\
\hline $\begin{array}{l}\text { Kumera et al., } \\
2018[37]\end{array}$ & $\begin{array}{l}\text { January to } \\
\text { February } \\
2016\end{array}$ & $\begin{array}{l}\text { cross- } \\
\text { sectional }\end{array}$ & $\begin{array}{l}\text { Institution } \\
\text { based }\end{array}$ & $\begin{array}{l}\text { University of } \\
\text { Gondar Hospital, } \\
\text { Amhara }\end{array}$ & Both & 402 & 126 & 31.3 & $\begin{array}{l}\text { Hookworm, E. histolytica } \\
\text { A. lumbricoides, H. nana } \\
\text { G. lamblia, S. stercoralis, S. mansoni, Tanea } \\
\text { species }\end{array}$ \\
\hline $\begin{array}{l}\text { Tulu et al., } 2019 \\
{[11]}\end{array}$ & $\begin{array}{l}\text { September } 7 \\
\text { to October } \\
25,2017\end{array}$ & $\begin{array}{l}\text { case } \\
\text { control }\end{array}$ & $\begin{array}{l}\text { Institution } \\
\text { based }\end{array}$ & $\begin{array}{l}\text { Health facilities } \\
\text { in Horo } \\
\text { Guduru, Oromia }\end{array}$ & $\begin{array}{l}\text { formalin-ether } \\
\text { Concentration }\end{array}$ & 573 & 101 & 17.6 & $\begin{array}{l}\text { Hookworm, T. trichiura, H. nana, A. } \\
\text { lumbricoides }\end{array}$ \\
\hline $\begin{array}{l}\text { Gebrehiwet } \\
\text { et al., } 2019 \text { [31] }\end{array}$ & $\begin{array}{l}\text { Not } \\
\text { described }\end{array}$ & $\begin{array}{l}\text { cross- } \\
\text { sectional }\end{array}$ & $\begin{array}{l}\text { Institution } \\
\text { based }\end{array}$ & $\begin{array}{l}\text { Maytsebri } \\
\text { Hospital, Tigray }\end{array}$ & Kato-Katz & 448 & 229 & 51.1 & Hookworm, T. trichiura, A. lumbricoides \\
\hline $\begin{array}{l}\text { Hailu et al., } 2019 \\
\text { [33] }\end{array}$ & $\begin{array}{l}\text { February to } \\
\text { June, } 2017\end{array}$ & $\begin{array}{l}\text { cross- } \\
\text { sectional }\end{array}$ & $\begin{array}{l}\text { Institution } \\
\text { based }\end{array}$ & $\begin{array}{l}\text { Health centers in } \\
\text { W/Gojjam, } \\
\text { Amhara }\end{array}$ & $\begin{array}{l}\text { Formol-ether } \\
\text { concentration }\end{array}$ & 743 & 276 & 37.1 & $\begin{array}{l}\text { Hookworm, E. histolytica } \\
\text { G. lamblia, A. lumbricoides }\end{array}$ \\
\hline $\begin{array}{l}\text { Weldekidan } \\
\text { et al., } 2018 \text { [44] }\end{array}$ & $\begin{array}{l}\text { February } 16 \\
\text { to May } 8 \\
2017\end{array}$ & $\begin{array}{l}\text { case } \\
\text { control }\end{array}$ & $\begin{array}{l}\text { Institution } \\
\text { based }\end{array}$ & $\begin{array}{l}\text { Health facilities } \\
\text { in Durame, } \\
\text { SNNPR }\end{array}$ & $\begin{array}{l}\text { formalin-ether } \\
\text { Concentration }\end{array}$ & 333 & 92 & 25.2 & Hookworm, G. lamblia, A. lumbricoides \\
\hline $\begin{array}{l}\text { Gebreegziabiher } \\
\text { et al., } 2014 \text { [19] }\end{array}$ & $\begin{array}{l}\text { October } \\
2011 \text { to July } \\
2012\end{array}$ & $\begin{array}{l}\text { cross- } \\
\text { sectional }\end{array}$ & $\begin{array}{l}\text { Institution } \\
\text { based }\end{array}$ & $\begin{array}{l}\text { Mekele, Ayder, } \\
\text { Semen Heath } \\
\text { center, Tigray }\end{array}$ & $\begin{array}{l}\text { Direct wet } \\
\text { mount }\end{array}$ & 85 & 30 & 35.3 & $\begin{array}{l}\text { T. trichiura, E. histolytica } \\
\text { A. lumbricoides, H. nana } \\
\text { G. lamblia, S. mansoni, E. vermicularis }\end{array}$ \\
\hline
\end{tabular}

\section{Quality assessment}

Three authors, (ZA, AM and HD), rated the quality of included studies utilizing Newcastle - Ottawa Scale which enables to assess the quality of each article by their methodological merit, comparability caliber and outcome excellence [18]. In due process, arguments between the authors were settled through in-depth discussion and articles were included upon consensus.

\section{Statistical analysis}

The extracted data were transferred to STATA software version 14.0 to analyze the pooled prevalence of IPIs and odds ratios of the associated factors. Heterogeneity among included studies was assessed using percentage of variance $\left(\mathrm{I}^{2}\right)$ and P-values. Random effect model was employed to estimate the pooled prevalence of intestinal parasites and summary odds ratios of factors associated with infections.

Begg's rank test and Egger's regression intercept tests were also carried out to indicate the correlation between the effect sizes and sampling variance in order to determine publication bias.

Subgroup analysis was done based on study region, types of parasites (helminthic, protozoa or both species), technique of stool examination (formalin-ether concentration, direct wet mount, or both and others) and study setting (institution or community based).

\section{Protocols and registration}

This systematic review and Meta-analysis is registered on PROSPERO under a registration number of CRD42020189115 and can be accessed at https://www. crd.york.ac.uk/PROSPERO.

\section{Results}

Characteristics of the included articles

A total of 31 studies were included in this systematic review and meta-analysis. The number of pregnant women participated in the studies ranged from 85 to $783[19,20]$ constituting a total population of 12,118 . The reported prevalence of IPIs was ranging from $7.3 \%[21,22]$ to $76.0 \%$ [20]. Twenty nine studies [4, $5,10,12,19-43]$ were cross-sectional while the rest two were case-controls [11, 44]. The studies were published from 2013 up to 2020. Regarding geographical distribution, 10 studies were reported from Amhara region $[4,10,20,25,33,34,36-38,40], 8$ from SNNPR [23, 26-29, 42-44], 8 from Oromia [5, 11, 12, 24, 32, 35, 39, 41], 4 from Tigray [19, 22, 30, 31] and One from Addis Ababa [21] (Table 1). 


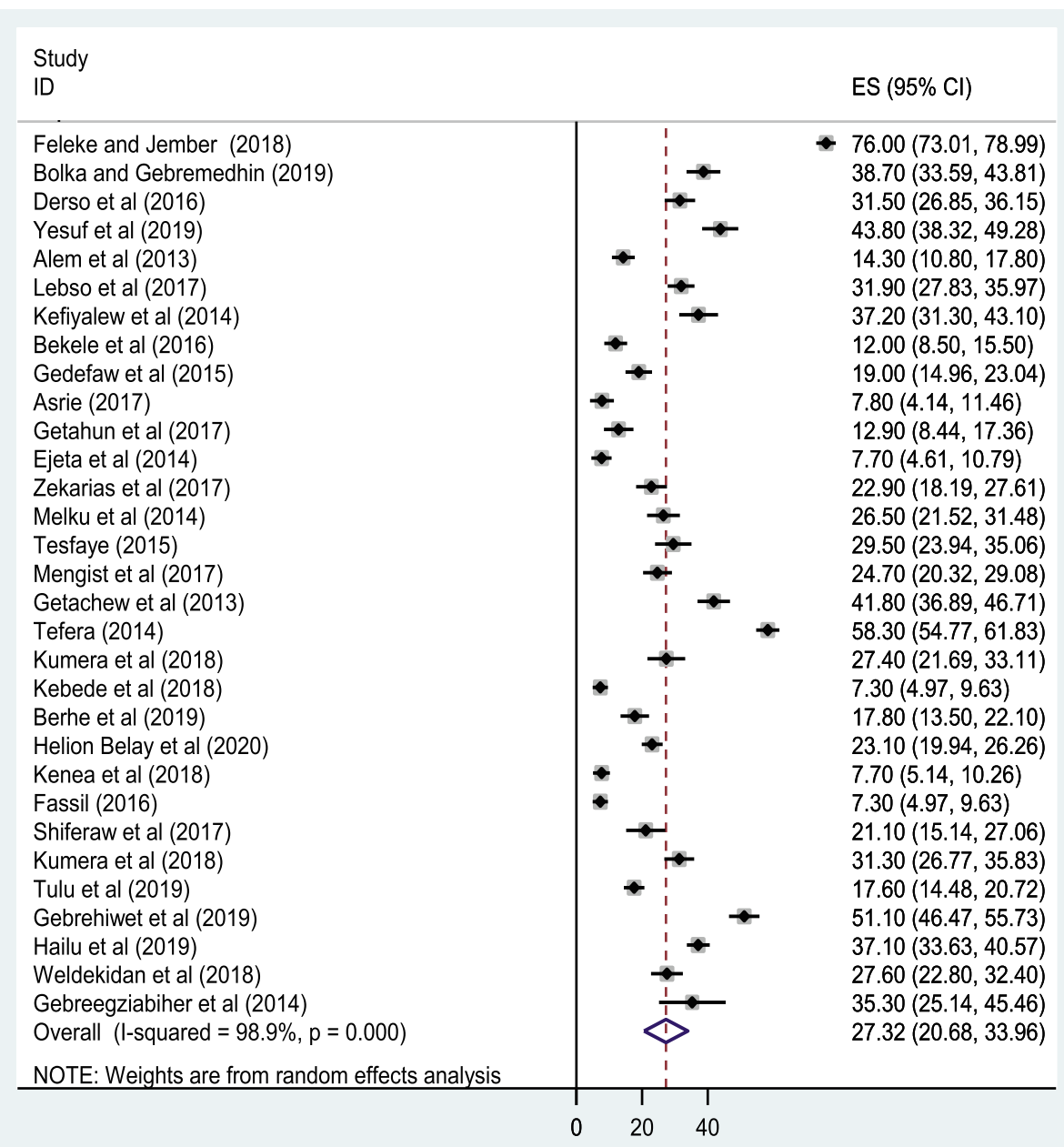

Fig. 2 Forest plot of the pooled prevalence of IPIs among pregnant mothers in Ethiopia

Pooled prevalence of intestinal parasitic infection among pregnant women in Ethiopia

The estimated pooled prevalence of IPIs among pregnant women in Ethiopia is $27.32 \%$ (95\% CI: 20.61, 33.87; $\mathrm{I}^{2}=98.9 \% p=<0.001$ ) (Fig. 2). Statistically significant heterogeneity was observed in the estimation of this pooled prevalence. Begg's rank and Egger's regression tests were carried out to statistically determine publication bias [45]. Based on the results, absence of significant publication bias was declared objectively with $P=0.068$ and $P=0.063$, consecutively.

\section{IPIs and anemia}

Among the included articles 16 of them [5, 10-13, 21, $22,25,26,28,33,35,37,38,41,44]$ reported that IPIs are significantly associated with anemia during pregnancy while 4 studies [27, 29, 34, 43] revealed that there is no association between occurrence of anemia among pregnant mothers who contracted IPIs. A single study disclosed that IPIs during pregnancy are significantly associated with maternal under-nutrition [36].

\section{Types of intestinal parasite}

In this study, the most prevalent type of intestinal parasite identified was Hookworm followed by Ascaris lumbricoides with a prevalence of $11.12 \%$ (95 \%CI: 8.21, 14.02) and 10.34 (95 \%CI: 7.09, 13.59); respectively. The least reported parasites are Enterobius vermicularis and Taenia species (Table 2).

\section{Subgroup analysis}

A subgroup analysis based on study regions revealed that Oromia had the highest prevalence estimate accounting 29.78 \% (95 \% CI: 15.97, 43.60) closely followed by Amhara region $29.63 \%$ (95\% CI: $15.37,43.89)$, Tigray region $27.74 \%$ (95\% CI: 6.56, 48.93) and SNNPR $24.23 \%$ 
Table 2 Pattern of Intestinal parasites among pregnant women

\begin{tabular}{|c|c|c|c|c|c|}
\hline Types of parasites & Species of parasite & No of studies & $\begin{array}{l}\text { Pooled prevalence } \\
(95 \% \mathrm{Cl})\end{array}$ & $\mathrm{I}^{2}$ & P-values \\
\hline \multirow[t]{8}{*}{ Helminthic } & Hookworm & 22 & $11.2 \%(8.21,14.02)$ & $97.3 \%$ & $<0.001$ \\
\hline & Ascaris lumbricoides & 20 & $10.34 \%(7.09,13.59)$ & $97.9 \%$ & $<0.001$ \\
\hline & Schistosomia mansoni & 10 & $3.42 \%(1.82,5.01)$ & $95.3 \%$ & $<0.001$ \\
\hline & Strongyloides stercolaris & 5 & $1.56 \%(0.31,2.82)$ & $91.7 \%$ & $<0.001$ \\
\hline & Trichuris trichiura & 11 & $2.85 \%(1.76,3.94)$ & $84.3 \%$ & $<0.001$ \\
\hline & Hymenolepis nana & 8 & $1.09 \%(0.56,1.63)$ & $50.8 \%$ & 0.047 \\
\hline & Taenia species & 7 & $0.94 \%(0.57,1.31)$ & $65.4 \%$ & 0.022 \\
\hline & Enterobius vermicularis & 4 & $0.81 \%(0.02,1.63)$ & 58.31 & 0.031 \\
\hline \multirow[t]{2}{*}{ Protozoa } & Giardia lamblia & 13 & $5.25 \%(3.41,7.09)$ & $89.5 \%$ & $<0.001$ \\
\hline & Entamoeba histolytica & 11 & $6.89 \%(4.07,9.71)$ & $94.0 \%$ & $<0.001$ \\
\hline Mixed & Mixed infection & 11 & $7.08 \%(4.18,9.98)$ & $95.6 \%$ & $<0.001$ \\
\hline
\end{tabular}

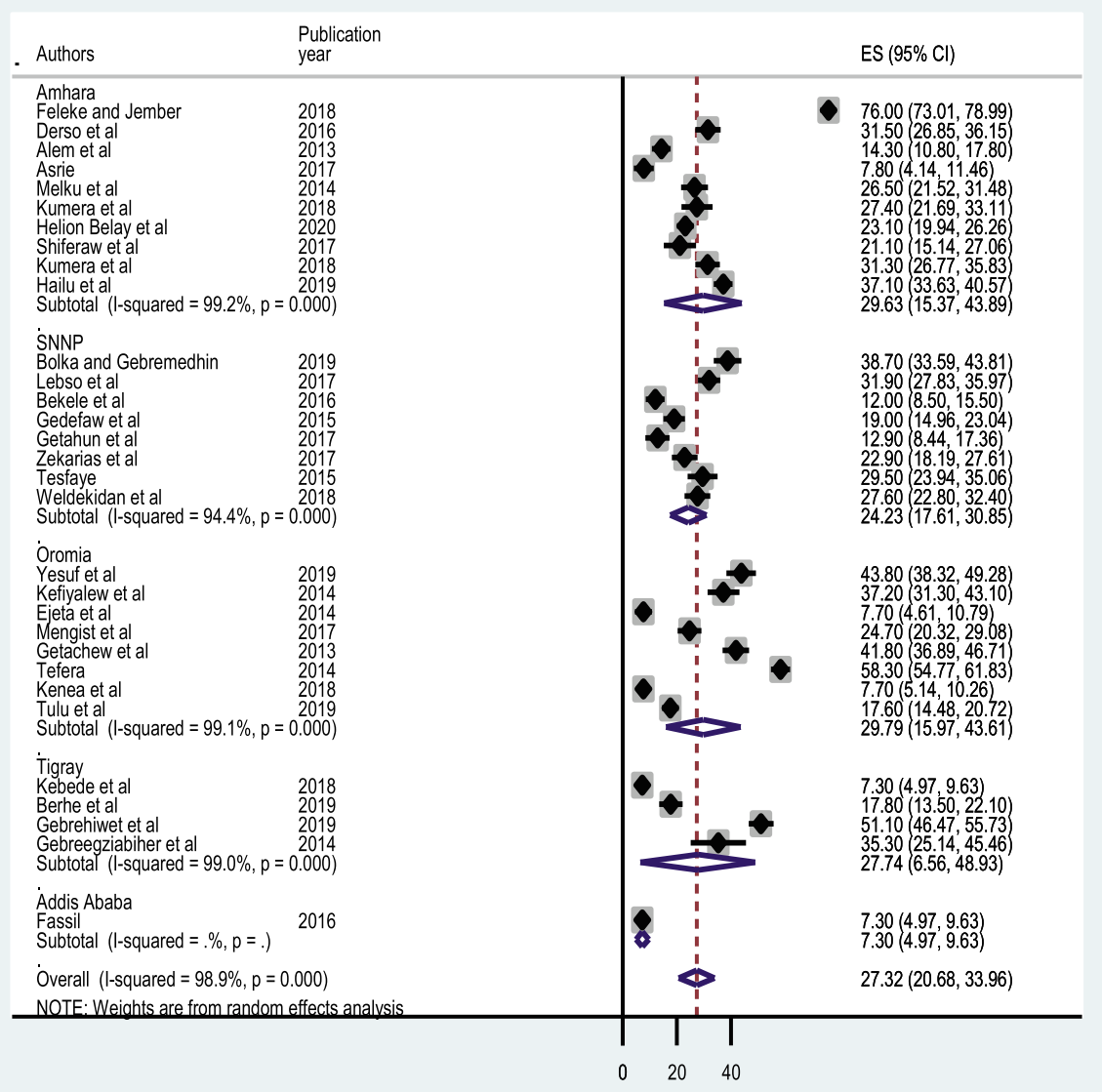

Fig. 3 Forest plot of subgroup analysis based on regions 


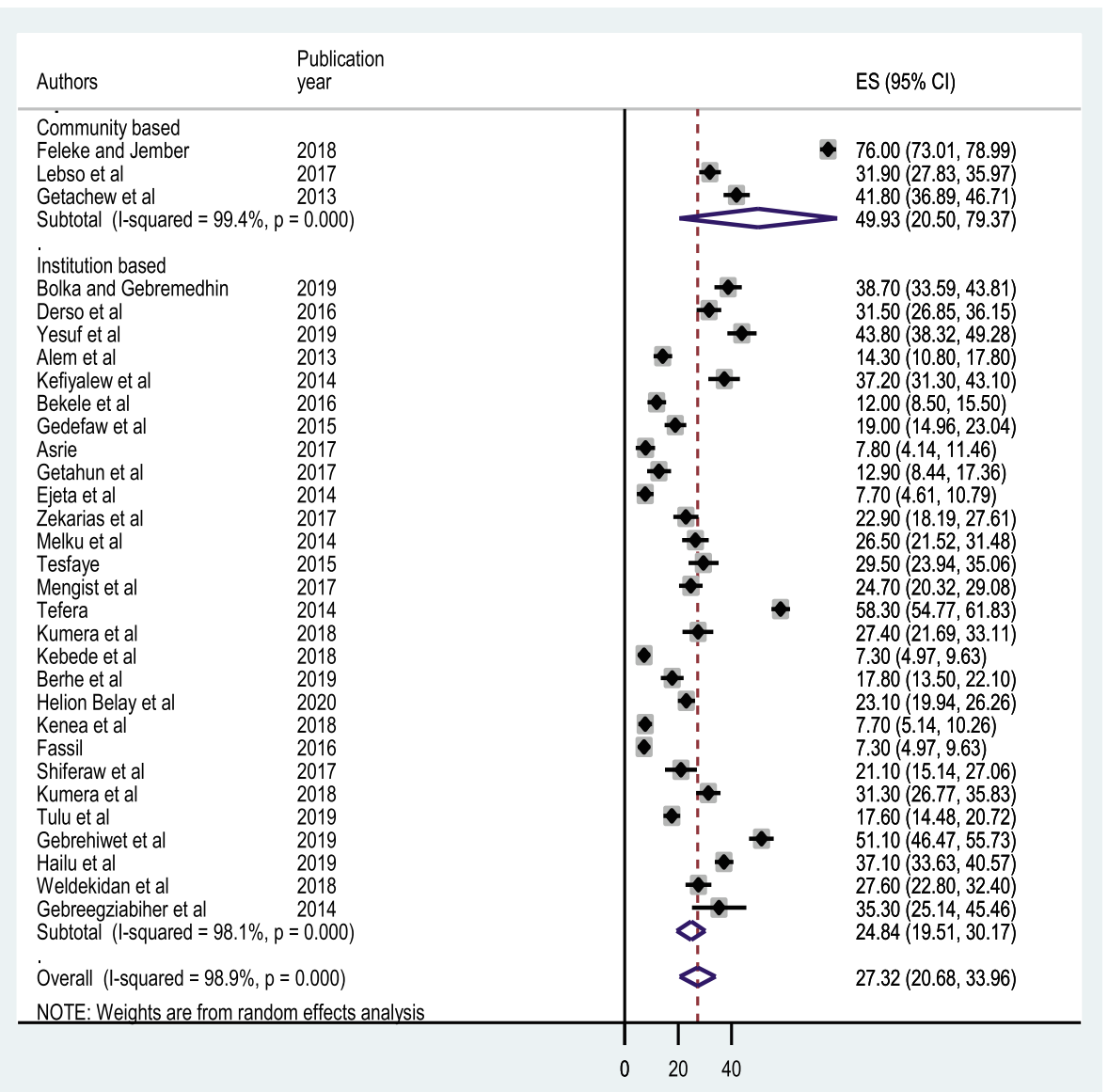

Fig. 4 Forest plot of subgroup analysis based on study setting

(95\% CI: 17.61, 30.85) (Fig. 3). Another subgroup analysis by study setting showed higher prevalence in studies done in the community than studies done institutions with a pooled prevalence of $49.93 \%$ (95 \%CI: $20.49,79.37)$ and $24.84 \%$ (95\%CI: 19.51, 30.17); respectively (Fig. 4). A similar analysis depending on the technique of stool examination indicates a combination of formalin-ether concentration and direct wet mount reported a higher prevalence of IPIs (35.99\%; $95 \%$ CI: $26.22,45.78)$ than studies that used a single stool examination to diagnose intestinal parasites (Fig. 5).

\section{Factors associated with intestinal parasitic infection among pregnant women in Ethiopia}

Our analysis identified that residence area, being bare footed, hand washing habit and eating uncooked/raw vegetables have significant association with the occurrence of IPIs among pregnant women. Pregnant women from rural areas were 6.3 more likely to develop IPIs when compared to urban dweller pregnant mothers $(\mathrm{OR}=6.31 ; 95 \%$ CI: 20, 32.99; $P=0.002$ ) (Fig. 6 A).
Likewise, a barefooted women were 2.79 times more likely to be infected with IPIs than those who wore shoes $(\mathrm{OR}=2.79$; $95 \%$ CI: 1.82, 9.48; $P=0.01$ ) (Fig. 6B). Similarly; pregnant women who had no hand washing habit and who consumed uncooked/raw vegetables were more likely to be infected with intestinal parasites compared to their counterparts $(\mathrm{OR}=3.02 ; 95 \% \mathrm{CI}: 1.64,14.33$; $P<0.001)$ (Fig. $6 \mathrm{C})$ and $(\mathrm{OR}=1.24$; $95 \% \mathrm{CI}: 1.65,2.37$; $P<0.001$ ) (Fig. 6 C); respectively.

\section{Discussion}

Women living in low-income countries are at a higher risk of acquiring IPIs that leads to severe anemia. This scenario will put both the mother and the baby at higher risk of morbidity and mortality. This study was conducted to summarize the current evidence of IPIs and associated factors among pregnant women in Ethiopia.

31 eligible studies that have quantified the magnitude of IPIs were included. Accordingly, the pooled prevalence of IPIs among pregnant women in Ethiopia was estimated to be $27.32 \%$. However, this pooled prevalence 


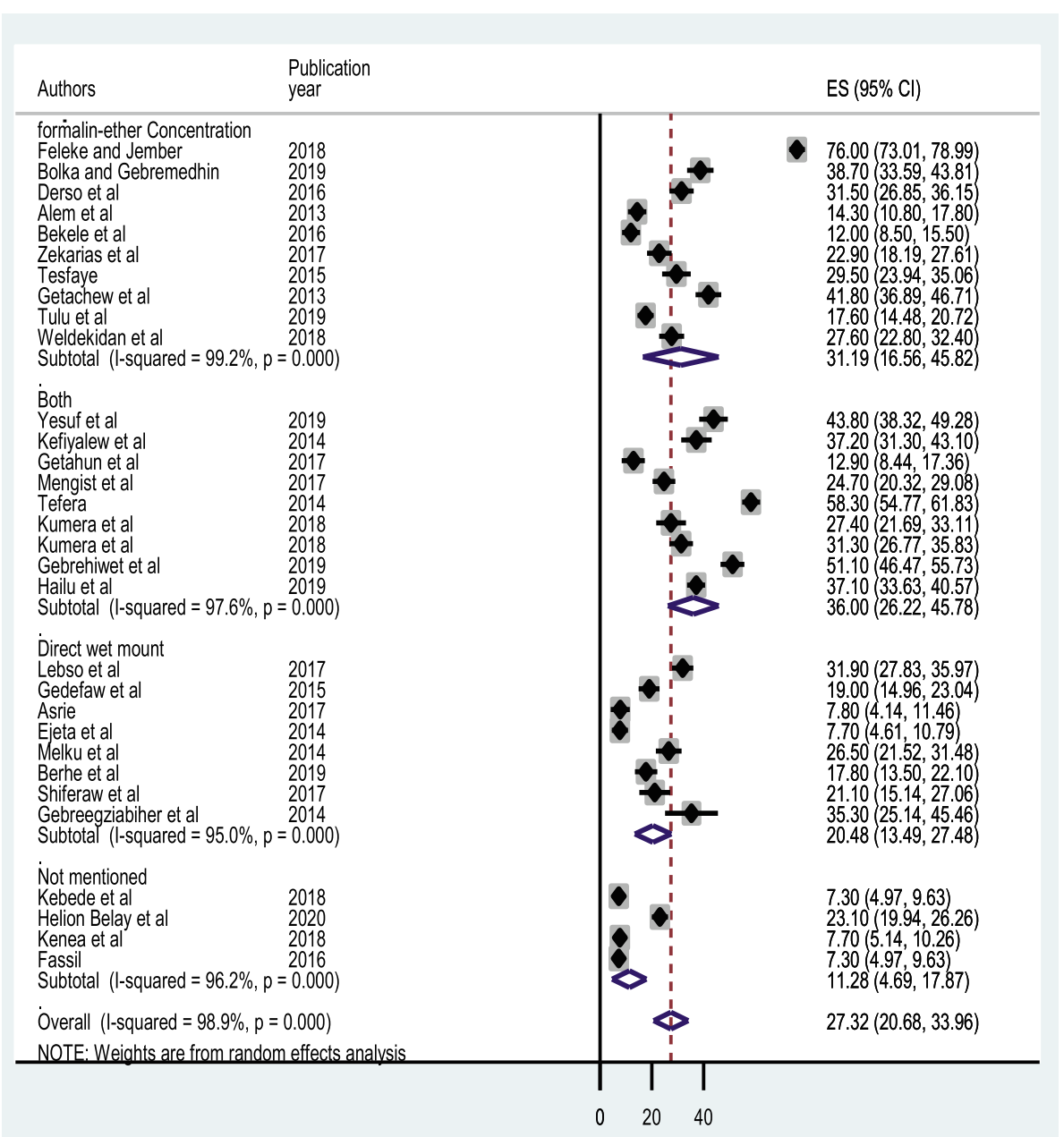

Fig. 5 Forest plot of subgroup analysis based on techniques of stool examination

is less than studies from Brazil, where $57.1 \%$ pregnant women were harbored at least one parasite [46], Cameroon (31.91\%) [47],India (42.67\%) [48] and Colombia (41\%) [49].

But, our finding is higher than reports from Kenya (24 and $13.8 \%$ ) [50, 51], Ghana (23.0\%) [52], Nigeria (20.8\%) [53], Sudan (13.0\%) [54] and other multicounty studies (20\%) [55]. These differences might be attributed to socioeconomic status, poor hygiene and sanitary facilities, weather, climate and environmental factors in the countries. It is also estimated that more than one-third of Sub-Sahara population are infected with at least one species of helminths [56]. Though pregnant women are vulnerable to IPIs, being pregnant was not yet regarded as a significant risk factor for acquiring enteric parasitic infections in Benin [57].

Hookworm and Ascariasis infection were the most prevalent in this review. Similarly, a global systematic review and meta-analysis identified Hookworm and Ascaris lumbricoides as the leading helminths affecting pregnant women while Giardia lamblia and Entamoeba histolytica lead protozal infection [16]. The most prevalent types of intestinal parasite identified among pregnant women were Ascaris lumbricoides followed and Hookworm in Kenya and Benin [51, 57]. Another study in Kenya identified Hookworm as one of the most common infestation at the first ANC visit [50]. Giardia lamblia and Ascaris lumbricoides in Colombia [49], Schistosoma mansoni and Trichuris Trichiura in Ghana [58] were the most prevalent parasitic infections. Trichuris trichiura was reported as a predominant parasite followed by $A$. lumbricoides in other study [59] and A. lumbricoides was dominantly identified parasites followed by $T$. trichiura Venezuela [60].

In addition, our subgroup analysis indicated that the rates of IPIs among pregnant women varied across different regions of the country, study settings and technique of stool examination. As a result, the prevalence is higher in Oromia and Amhara regional state. 


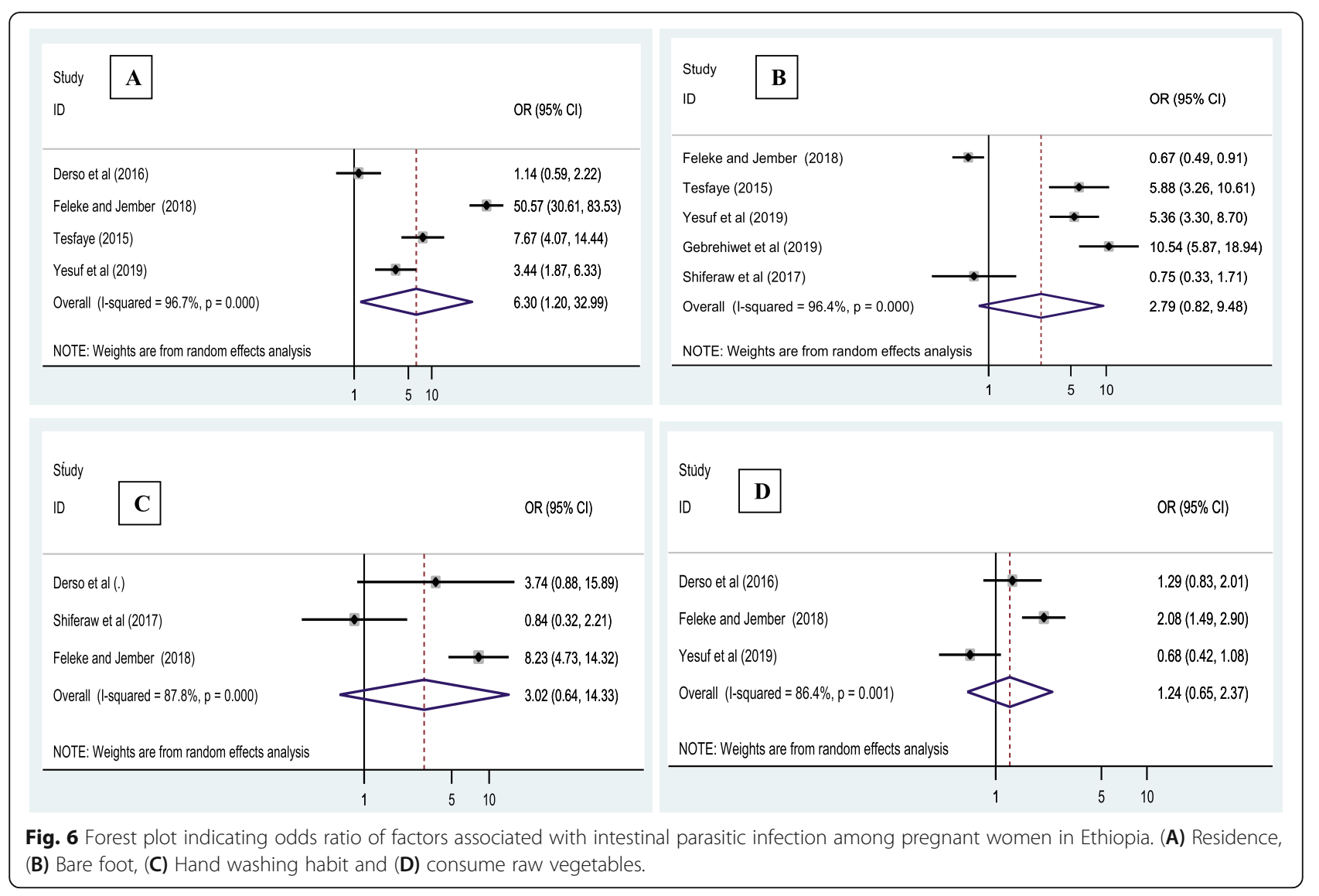

Studies conducted in the community showed a higher prevalence than studies done in institutions with a pooled prevalence of $49.93 \%$ (95 \%CI: 20.49, 79.37) and $24.84 \%$ (95 \%CI: 19.51, 30.17); respectively. This might be related with inadequate water supply and poor sanitation [61]. On the other hand, studies that used a combination of formalin-ether concentration and direct wet mount stool examination techniques reported a higher prevalence of IPIs (35.99\%; $95 \% \mathrm{CI}: 26.22,45.78$ ) than studies that used a single stool examination to diagnose intestinal parasites. This evidenced that the detection rate of intestinal parasites improved by concentrating stool samples [39].

Different factors for IPIs were analyzed and the pooled odds ratio was summarized. As a result, rural residents, being bare footed, poor hand washing habits and eating uncooked/raw vegetables have significant association with the occurrence of IPIs among pregnant women in Ethiopia. Similarly, the high occurrence of parasitic infection has been related to the poor hygiene condition [46] and residence area [16, 47] in other studies. It is also evidenced that factors influencing the continuous transmission of IPIs in sub-Saharan countries include poor sanitation and hygiene and non-availability of potable water for domestic use [53]. In line with our analysis, pregnant women who practiced hand washing regularly had lesser infection in India, Kenya and Benin $[48,51,57]$.

Contradicting to this view, the prevalence of intestinal parasites was almost same in both rural and urban pregnant women in India [48]. The finding of this review was consistent with studies in Benin where pregnant women who consume uncooked/raw vegetables from food vendors were more likely to be infected with intestinal parasites. However, in contrary to our review result; being barefooted was not significantly affect the prevalence of IPIs [57]. Consuming unwashed vegetable and being barefooted were associated with IPIs among pregnant women [59].

In line with a review conducted in Sub-Saharan countries [53, 56], we also found that the odds of IPIs were found to be higher in pregnant mothers living in rural areas. Because, place of residence, usually determines people's lifestyles, income, social and cultural activities, and most notably their health conditions.

\section{Strengths and limitations}

Our meta-analysis tried to elucidate a national figure on prevalence of IPIs during pregnancy. It included studies 
done both at institution and community settings. Both cros-sectional and case control stud designs were included which enabled us to identify temporal relationship among predictors and outcome variables. As per our search, this is the first analysis done in Ethiopia. However, this meta-analysis is done only on 5 regions of Ethiopia and as a result; the whole image of the problem might be under represented.

\section{Conclusions}

Our systematic review and meta-analysis has estimated a high prevalence of IPIs during pregnancy in Ethiopia. The result indicates the need for priority interventions targeted to improve maternal health during pregnancy. Investing in maternal health is also a key strategy to reduce low birth weight and preterm birth. Apart from socio- economic factors, IPIs during pregnancy are related with poor hygienic practices, being barefooted and eating habits of raw vegetables. Therefore, an optimal personal hygiene and developing shoes wearing habit by the mothers is essential to meet both maternal and child requirements and reduce adverse health consequences in addition to spaced pregnancy. To prevent anemia, pregnant women are advised to take iron, folate supplements, eat iron-rich foods, and prevent intestinal worms.

\section{Abbreviations}

OR: odds ratio; Cl: confidence interval; IPIs: Intestinal parasitic infections; IUGR: Intrautrine growth retardation; STH: soil-transmitted helminths; PC: preventive chemotherapy; PRISMA: preferred reporting items for systematic review and meta analysis; SNNPR: southern national, nationalities and peoples region; ANC: antenatal care

\section{Acknowledgements}

We would like to acknowledge all academic staffs of Addis Ababa University, Debre Tabor University and Bahir Dar University who helped us in this particular study.

\section{Authors' contributions}

ZA responsible for design, article selection, data extraction, statistical analysis and manuscript drafting. AM, HD, GS were involved in selection of articles, statistical analysis and manuscript editing. AA reviewed the protocols, tools, analysis and manuscript editing. All the authors read and approved the final draft of the manuscript.

\section{Funding}

'No funding was obtained for this study'.

\section{Availability of data and materials}

The datasets used during the current study are available at the corresponding author upon reasonable request.

\section{Declarations}

\section{Ethics approval and consent to participate}

'Not applicable'.

\section{Consent for publication}

'Not applicable'.

\section{Competing interest}

'The authors declare that they have no competing interests'.

\section{Author details}

'Department of Biomedical Sciences, College of Health Sciences, Debre Tabor University, Debre Tabor, Ethiopia. ${ }^{2}$ Department of Medical Microbiology, College of Health Sciences, Bahir Dar University, Bahir Dar, Ethiopia. ${ }^{3}$ Department of Public Health, College of Health Sciences, Debre Tabor University, Debre Tabor, Ethiopia. ${ }^{4}$ Department of Anatomy, School of Medicine, College of Health Sciences, Addis Ababa University, Addis Ababa, Ethiopia. ${ }^{5}$ Traditional and Modern Medicine Research Directorate, Ethiopian Public Health Institute, Addis Ababa, Ethiopia.

Received: 1 November 2020 Accepted: 28 May 2021

Published online: 01 July 2021

\section{References}

1. Alfonso J. Rodr'iguez-Morales RABea. Intestinal Parasitic Infections among pregnant women in Venzuela. Infectious Diseases in Obstetrics Gynecology. 2006;2006:1-5.

2. Nipurte Roopal PS, Koticha Avani N, Gita, Mehta Preeti. Correlation of sociodemographic factors and intestinal parasites in pregnant women. Int J Res Med Sci. 2020;8(1):244-51.

3. H. MPAIRWE RTAE. Pregnancy and helminth infections. Parasite Immunol. 2014;36:328-37.

4. Derso A, Nibret E, Munshea A. Prevalence of intestinal parasitic infections and associated risk factors among pregnant women attending antenatal care center at Felege Hiwot Referral Hospital, northwest Ethiopia. BMC Infect Dis. 2016;16(1):530.

5. Ejeta E, Alemnew B, Fikadu A, Fikadu M, Tesfaye L, Birhanu T, et al. Prevalence of anaemia in pregnant womens and associated risk factors in Western Ethiopia. Food Sci Qual Manag. 2014;31(6):82-91.

6. Susana Vaz Nery AJP, Ebba, Abate, et al The role of water, sanitation and hygiene interventions in reducing soil-transmitted helminths: interpreting the evidence and identifying next steps. Parasites Vectors. 2019;12(273):1-8.

7. Akinbo FOOT, Okaka CE, Oriakhi MO. Co-infection of malaria and intestinal parasites among pregnant women in Edo State, Nigeria. J Med Trop. 2017; 19:43-8.

8. Nigatu Tuasha1 EH, Berhanu Erko and Beyene Petros. Comorbidity of intestinal helminthiases among malaria outpatients of Wondo Genet health centers, southern Ethiopia. BMC Infectious Diseases. 2019;19(659):1-8.

9. Ntombela JMT-GaNP. A Double Load to Carry: Parasites and Pregnancy. South Afr J Infect Dis. 2014;29(2):52-5

10. Asrie F. Prevalence of anemia and its associated factors among pregnant women receiving antenatal care at Aymiba Health Center, northwest Ethiopia. Journal of blood medicine. 2017;8:35.

11. Tulu BD, Atomssa EM, Mengist HM. Determinants of anemia among pregnant women attending antenatal care in Horo Guduru Wollega Zone, West Ethiopia: Unmatched case-control study. PloS one. 2019;14(10): e0224514.

12. Kefiyalew F, Zemene E, Asres Y, Gedefaw L. Anemia among pregnant women in Southeast Ethiopia: prevalence, severity and associated risk factors. BMC Res Notes. 2014;7(1):771.

13. Mengist HM, Zewdie $\mathrm{O}$, Belew A. Intestinal helminthic infection and anemia among pregnant women attending ante-natal care (ANC) in East Wollega, Oromia, Ethiopia. BMC Res Notes. 2017;10(1):440.

14. Samuel Crowther Kofi Tay EANaWW. Parasitic infections and maternal anaemia among expectant mothers in the Dangme East District of Ghana. BMC Res Notes. 2017;10(3):1-9.

15. Hopkins CH. Pregnant with Parasites: Assessing Parasitic Infections in Pregnant Women of the Kwale District. Independent Study Project (ISP) Collection, 1491. 2013.

16. Taghipour A, Ghodsian S, Jabbari M, Olfatifar M, Abdoli A, Ghaffarifar F. Global prevalence of intestinal parasitic infections and associated risk factors in pregnant women: a systematic review and meta-analysis. Transactions of the Royal Society of Tropical Medicine and Hygiene. 2020.

17. WHO. 2030 targets for soil-transmitted helminthiases control programmes. Geneva: World Health Organization. CC BY-NC-SA 3.0 IGO. Neglected tropical diseases. 2019.

18. Luchini C, Stubbs B, Solmi M, Veronese N. Assessing the quality of studies in meta-analysis: advantages and limitations of the Newcastle Ottawa Scale. World J Meta-Anal. 2017;5:1-48. 
19. Gebreegziabiher D, Desta K, Desalegn G, Howe R, Abebe M. The effect of maternal helminth infection on maternal and neonatal immune function and immunity to tuberculosis. PloS one. 2014;9(4):1-7.

20. Feleke BE, Jember TH. Prevalence of helminthic infections and determinant factors among pregnant women in Mecha district, Northwest Ethiopia: a cross sectional study. BMC Infect Dis. 2018;18(1):373.

21. Fassil R. Prevalence of Anemia Among Pregnant Women Attending Antenatal Care at Selam Health Center. Addis Ababa: Addis Ababa University; 2016

22. Kebede A, Gerensea H, Amare F, Tesfay Y, Teklay G. The magnitude of anemia and associated factors among pregnant women attending public institutions of Shire Town, Shire, Tigray, Ethiopia N. 2018. BMC research notes. 2018;11(1):1-6.

23. Bolka A, Gebremedhin S. Prevalence of intestinal parasitic infection and its association with anemia among pregnant women in Wondo Genet district, Southern Ethiopia: a cross-sectional study. BMC Infect Dis. 2019;19(1):483.

24. Yesuf DA, Abdissa LT, Gerbi EA, Tola EK. Prevalence of intestinal parasitic infection and associated factors among pregnant women attending antenatal care at public health facilities in Lalo Kile district, Oromia, Western Ethiopia. BMC Res Notes. 2019;12(1):735

25. Alem M, Enawgaw B, Gelaw A, Kena T, Seid M, Olkeba Y. Prevalence of anemia and associated risk factors among pregnant women attending antenatal care in Azezo Health Center Gondar town, Northwest Ethiopia. 2013.

26. Lebso M, Anato A. Loha3 E. Prevalence of anemia and associated factors among pregnant women in Southern Ethiopia: A community based crosssectional study. PloS one. 2017;12(12):1-11.

27. Bekele A, Tilahun M, Mekuria A. Prevalence of anemia and its associated factors among pregnant women attending antenatal care in health institutions of Arba Minch Town, Gamo Gofa Zone, Ethiopia: a crosssectional study. Anemia. 2016;2016.

28. Gedefaw L, Ayele A, Asres Y, Mossie A. Anaemia and associated factors among pregnant women attending antenatal care clinic in Walayita Sodo town, Southern Ethiopia. Ethiopian journal of health sciences. 2015;25(2): 155-64.

29. Getahun W, Belachew T, Wolide AD. Burden and associated factors of anemia among pregnant women attending antenatal care in southern Ethiopia: cross sectional study. BMC Res Notes. 2017;10(1):276.

30. Berhe B, Mardu F, Legese H, Gebrewahd A, Gebremariam G, Tesfay K, et al. Prevalence of anemia and associated factors among pregnant women in Adigrat General Hospital, Tigrai, northern Ethiopia, 2018. BMC Res Notes. 2019:12(1):310

31. Gebrehiwet MG, Medhaniye AA, Alema HB. Prevalence and associated factors of soil transmitted helminthes among pregnant women attending antenatal care in Maytsebri primary hospital, North Ethiopia. BMC Res Notes. 2019;12(1):644

32. Getachew M, Tafess K, Zeynudin A, Yewhalaw D. Prevalence Soi Transmitted Helminthiasis and malaria co-infection among pregnant women and risk factors in Gilgel Gibe dam Area, Southwest Ethiopia. BMC Res Notes. 2013;6(1):263.

33. Hailu T, Kassa S, Abera B, Mulu W, Genanew A. Determinant factors of anaemia among pregnant women attending antenatal care clinic in Northwest Ethiopia. Tropical diseases, travel medicine and vaccines. 2019; 5(1):13.

34. Helion Belay AM, Tariku A, Woreta SA, Demissie GD, Asrade G. Anemia and Associated Factors among Pregnant Women Attending Prenatal Care in Rural Dembia District, North West Ethiopia: A cross-Sectional Study. Ecol Food Nutr. 2020:59(2):154-74

35. Kenea A, Negash E, Bacha L, Wakgari N. Magnitude of Anemia and Associated Factors among Pregnant Women Attending Antenatal Care in Public Hospitals of Ilu Abba Bora Zone, South West Ethiopia: A CrossSectional Study. Anemia. 2018;2018.

36. Kumera G, Gedle D, Alebel A, Feyera F, Eshetie S. Undernutrition and its association with socio-demographic, anemia and intestinal parasitic infection among pregnant women attending antenatal care at the University of Gondar Hospital, Northwest Ethiopia. Maternal health neonatology perinatology. 2018;4(1):18.

37. Kumera $\mathrm{G}$, Haile $\mathrm{K}$, Abebe N, Marie $\mathrm{T}$, Eshete $\mathrm{T}$. Anemia and its association with coffee consumption and hookworm infection among pregnant women attending antenatal care at Debre Markos Referral Hospital, Northwest Ethiopia. PloS one. 2018;13(11):1-14.
38. Melku M, Addis Z, Alem M, Enawgaw B. Prevalence and predictors of maternal anemia during pregnancy in Gondar, Northwest Ethiopia: an institutional based cross-sectional study. Anemia. 2014;2014:1-9.

39. Mengist HM, Demeke G, Zewdie O, Belew A. Diagnostic performance of direct wet mount microscopy in detecting intestinal helminths among pregnant women attending ante-natal care (ANC) in East Wollega, Oromia, Ethiopia. BMC Res Notes. 2018;11(1):276.

40. Shiferaw MB, Zegeye AM, Mengistu AD. Helminth infections and practice of prevention and control measures among pregnant women attending antenatal care at Anbesame health center, Northwest Ethiopia. BMC Res Notes. 2017;10(1):274.

41. Tefera G. Determinants of anemia in pregnant women with emphasis on intestinal helminthic infection at Sher-Ethiopia Hospital, Ziway, Southern Ethiopia. Immunol Infect Dis. 2014;2(4):33-9.

42. Tesfaye DJ. Prevalence of intestinal helminthiases and associated factors among pregnant women attending antenatal clinic of Nigist Eleni Mohammed memorial hospital, Hossana, Southern Ethiopia. Open Access Library Journal. 2015;2(07):1.

43. Zekarias B, Meleko A, Hayder A, Nigatu A, Yetagessu T. Prevalence of anemia and its associated factors among pregnant women attending antenatal care (ANC) in Mizan Tepi University Teaching Hospital, South West Ethiopia. Health Science Journal. 2017;11(5):1-8.

44. Weldekidan F, Kote M, Girma M, Boti N, Gultie T. Determinants of anemia among pregnant women attending antenatal clinic in public health facilities at Durame Town: unmatched case control study. Anemia. 2018; 2018.

45. Lin L, Chu H. Quantifying publication bias in meta-analysis. Biometrics. 2018; 74(3):785-94.

46. Maria Celia Dos Santos JMC-C, Carvalho-Neto,Marcus C. M.R.LIMA And Marisa Cristina. Enteric Parasites and Commensal in Pregnant Women Seen at the University Hospital Federal University of Uberlandia, State of Minas Gerais, Brazil. 1998;40(3):193-5.

47. Calvin Tonga CNB. Flore Chanceline Tchanga, Jacqueline Félicité Yengue, Godlove Bunda Wepnje, Hervé Nyabeyeu Nyabeyeu, Lafortune Kangam, Larissa Nono Kouodjip, Patrick Ntonga Akono, Léopold Gustave Lehman. Schistosomiasis among pregnant women in Njombe-Penja health district, Cameroon. J Infect Dev Ctries. 2019;13(12):1150-8.

48. Nipurte Roopal PS, Koticha, Avani. Nataraj Gita and Mehta Preeti. Correlation of sociodemographic factors and intestinal parasites in pregnant women. International Journal of Research in Medical Sciences. 2019;8(1):244-51.

49. Angela FE, Aranzales KR, Guenter Froeschl. Ángela María Pinzón Rondón and Maria Delius. Prevalence and risk factors for intestinal parasitic infections in pregnant women residing in three districts of Bogotá, Colombia. BMC Public Health. 2018;2018:1-15.

50. Elizabeth M. McClure SRM, Mungai P, Malhotra I, King CL, Goldenberg RL, Hudgens MG. Anna Maria Siega-Riz1, Arlene E. Dent. The Association of Parasitic Infections in Pregnancy and Maternal and Fetal Anemia: A Cohort Study in Coastal Kenya. PLOS Neglected Tropical Diseases. 2014;8(2):1-8.

51. Wekesa AW, Muleke CSM,Cl. and R. Odhiambo4. Intestinal Helminth Infections in Pregnant Women Attending Antenatal Clinic at Kitale District Hospital, Kenya. Journal of Parasitology Research. 2014;2014:1-5.

52. Godwin Fuseini DE. Bugre Gumah Kalifa and Dave Knight. Plasmodium and intestinal helminths distribution among pregnant women in the KassenaNankana District of Northern Ghana. Entomology and Nematology. 2010; 1(2):19-24.

53. Abiola Fatimah Adenowoa BEO. Bolajoko Idiat Ogunyinkaa and Abidemi Paul Kappoa. Impact of human schistosomiasis in sub-Saharan Africa. b r a z j in f e c t d i s 2015;19(2):196-205.

54. Abelrahman Khalid MAA, Abellatif Ashmaig AM, Ibrahim, Abdel-Aziz M, Ahmed I Adam. Schistosoma mansoni infection among prenatal attendees at a secondary-care hospital in central Sudan. International Journal of Gynecology and Obstetrics. 2012;116(2012).

55. WHO. Guideline: preventive chemotherapy to control soil-transmitted helminth infections in at-risk population groups. Geneva. Licence: CC BYNC-SA 30 IGO. 2017

56. Dadi HDDaAF. Burden and determinants of malnutrition among pregnant women in Africa: A systematic review and meta-analysis. PLOS ONE. 2019; 14(9):1-19.

57. Frederick Olusegun Akinbo EO. Adedapo Babatunde Anibaba Ande. Intestinal Nematodes Infection among Pregnant Women in Benin City. Nigerian Journal of Experimental and Clinical Biosciences. 2020;3(2):65-70. 
58. NANI EA. IMPACT OF SCHISTOSOMIASIS AND SOIL TRANSMITTED HELMINTH INFECTION AMONG PREGNANT. WOMEN IN THE DANGME EAST DISTRICT, GREATER ACCRA REGION. 2014.

59. Albert Njeru FMaSM. Status of Soil-transmitted helminthiasis among pregnant women attending antenatal clinic in Kilifi county hospital, Kenya. 2019.

60. Rodríguez-Morales AJ, Barbella RA, Case C, Arria M, Ravelo M, Perez H, et al. Intestinal parasitic infections among pregnant women in Venezuela. Infectious diseases in obstetrics and gynecology. 2006;2006.

61. Jeff rey Bethony SB, Marco Albonico, Stefan M Geiger, Alex Loukas, David Diemert, Peter J Hotez. <Soil-transmitted helminth infections: ascariasis, trichuriasis, and hookworm.pdf>. lancet. 2006;367:1521-32.

\section{Publisher's Note}

Springer Nature remains neutral with regard to jurisdictional claims in published maps and institutional affiliations.

Ready to submit your research? Choose BMC and benefit from:

- fast, convenient online submission

- thorough peer review by experienced researchers in your field

- rapid publication on acceptance

- support for research data, including large and complex data types

- gold Open Access which fosters wider collaboration and increased citations

- maximum visibility for your research: over $100 \mathrm{M}$ website views per year

At BMC, research is always in progress.

Learn more biomedcentral.com/submissions 